\title{
Rectified heat transfer into translating and pulsating vapor bubbles
}

\author{
Y. Hao and A. Prosperetti ${ }^{\mathrm{a})}$ \\ Department of Mechanical Engineering, The Johns Hopkins University, Baltimore, Maryland 21218
}

(Received 20 February 2002; revised 3 July 2002; accepted 1 August 2002)

\begin{abstract}
It is well known that, when a stationary vapor bubble is subject to a sufficiently intense acoustic field, it will grow by rectified heat transfer even in a subcooled liquid. The object of this paper is to study how translation, and the ensuing convective effects, influence this process. It is shown that, depending on the initial temperature distribution and other factors, convection can cause a destabilization of the bubble or its faster growth. Significant effects occur in parameter ranges readily encountered in practice. The phenomena described can therefore be exploited for bubble management, e.g., by increasing the condensation rate or promoting faster bubble growth and coalescence. In a saturated or a superheated liquid, heat rectification and convection reinforce each other and the bubble growth is accelerated by a translatory motion. () 2002 Acoustical Society of America. [DOI: 10.1121/1.1508789]

PACS numbers: 43.25.Yw [AJS]
\end{abstract}

\section{INTRODUCTION}

A vapor bubble subject to a sound field in a subcooled liquid exhibits the phenomenon of rectified heat transfer, by which it can either grow, or collapse at a slower rate than without sound (see, e.g., Wang, 1974; Khabeev, 1976; Akulichev et al., 1979; Patel et al., 1985; Gumerov, 2000; Hao and Prosperetti, 1999; Hao et al., 2001; Prosperetti and Hao, 2001, 2002). Similarly, in a superheated liquid, sound causes a faster growth than would occur in a constant pressure environment. The process is quite similar to the perhaps better known rectified diffusion of mass (see, e.g., Fyrillas and Szeri, 1994).

The physical origin of this behavior is due to the opposite effect of two competing mechanisms, which justifies the adjective "rectified" given to this phenomenon. When the bubble is compressed, some vapor condenses, the surface temperature rises, and heat is conducted away into the adjacent liquid. When the bubble expands during the following half cycle, evaporation causes a temperature drop of the bubble surface, with a consequent heat flux from the liquid. The net result of these opposing processes exhibits a bias toward an energy gain by the bubble because $(i)$ the spherical geometry forces a thicker boundary layer - and hence a smaller heat flux-during compression, and (ii) the surface area available for the phase change is bigger during expansion than during compression. ${ }^{1}$

In view of the reliance of these effects on heat transfer between the bubble and the liquid, it may be expected that they would be strongly influenced by a translational motion of the bubble. This expectation is borne out by the results of this paper, which presents a numerical investigation of the process under the simplifying assumption of a spherical bubble. Depending on conditions, in a subcooled liquid, we

\footnotetext{
a) Author to whom correspondence should be addressed; electronic mail: prosperetti@jhu.edu. Also with Department of Applied Physics, Twente Institute of Mechanics, and Burgerscentrum, University of Twente, AE 7500 Enschede, The Netherlands.
}

find that convection can either make the bubble more labile or increase its growth rate. The bubble condenses if it is abruptly exposed to cold liquid and the velocity is sufficiently large. A bubble which survives this initial stage, on the other hand, may grow faster than without translation. The explanation for this paradoxical finding is that, as explained before, the amount of heat transferred is greater during the expansion phase than the compression phase. During the expansion phase the bubble is cooler than its surroundings and, therefore, convection helps to increase its vapor content.

With this study we continue the investigation of the behavior of vapor bubbles under the joint action of acoustic and flow fields started in Hao and Prosperetti (1999) and continued in subsequent papers (Hao and Prosperetti, 2000; Hao et al., 2001; Prosperetti and Hao, 2001). This series of studies responds to the practical need of vapor bubble management under microgravity conditions, both to increase the critical heat flux in boiling and to control the void fraction of flowing two-phase cryogenic mixtures (Oka et al., 1992; Ervin et al., 1992; Sitter et al., 1998a, b).

\section{MATHEMATICAL FORMULATION AND NUMERICAL METHOD}

The mathematical model is based on our earlier work (Hao and Prosperetti, 1999, 2000); here we only present a summary of the formulation.

The bubble is assumed to remain spherical, with a radius $R(t)$ determined by a form of the Rayleigh-Plesset equation which accounts for weak liquid compressibility effects:

$$
\begin{aligned}
(1- & \left.\frac{\dot{R}}{c}\right) R \ddot{R}+\frac{3}{2}\left(1-\frac{\dot{R}}{3 c}\right) \dot{R}^{2} \\
& =\frac{1}{\rho}\left(1+\frac{\dot{R}}{c}+\frac{R}{c} \frac{d}{d t}\right)\left[p_{b}-P(t)\right] .
\end{aligned}
$$

Here dots denote time differentiation, $\rho$ and $c$ are the liquid density and speed of sound, $p_{b}$ is the pressure on the liquid 
side of the interface, and $P(t)$ is the ambient pressure which we will take in the form

$$
P(t)=P_{\infty} \pm P_{A} \sin \omega t,
$$

in which $P_{\infty}$ is the static pressure, $P_{A}$ is the acoustic pressure amplitude, and $\omega$ is the angular frequency of the sound field; the upper sign is chosen when the initial phase of the sound is compressive, and the lower one when it is expansive. It will be seen that the phase of the sound field is very important in the initial stages of the process. We assume the vapor pressure in the bubble $p_{V}$ to be spatially uniform and in saturated conditions with respect to the bubble surface temperature $T_{S}$; thus

$$
p_{V}=p_{\text {sat }}\left(T_{S}\right)=p_{b}+\frac{2 \sigma}{R}+4 \mu \frac{\dot{R}}{R},
$$

in which $\sigma$ and $\mu$ are the surface tension and viscosity coefficients; here and in the following we append the subscript $V$ to quantities pertaining to the vapor, and the subscript sat indicates quantities evaluated along the saturation line.

The energy equation in the liquid is

$$
\frac{\partial T}{\partial t}+\mathbf{u} \cdot \nabla T=D \nabla^{2} T
$$

where $T$ is the liquid temperature, $D$ is the liquid thermal diffusivity, and $\mathbf{u}$ is the liquid velocity field in the bubble rest frame. This equation is solved subject to the condition of undisturbed temperature far from the bubble, $T \rightarrow T_{\infty}$ for $r$ $\rightarrow \infty$ while, at the bubble surface, conservation of energy dictates that (Hao and Prosperetti, 1999)

$$
\left.4 \pi R^{2} k \frac{\partial T}{\partial r}\right|_{r=R(t)}=L \frac{d}{d t}\left(\frac{4}{3} \pi R^{3} \rho_{V}\right)+\frac{4}{3} \pi R^{3} \rho_{V} c_{s} \frac{d T_{S}}{d t} .
$$

Here $k$ and $L$ are the liquid thermal conductivity and latent heat, respectively, while $c_{s}=c_{p V}-L / T_{S}$ is the specific heat of the vapor along the saturation line; $c_{p V}$ is the vapor specific heat at constant pressure. For most of the calculations reported below, the initial temperature field equals $T_{\infty}$ everywhere, including the bubble surface.

We assume the bubble to move rectilinearly with velocity $U$ with respect to the liquid at rest at infinity. Keeping in mind the temperature dependence of viscosity, we find that typical Reynolds numbers in the cases we consider are of the order of several hundreds, which justifies the approximation of potential flow except possibly in the last stages of the total condensation of a bubble. Therefore we assume that, in the rest frame of the bubble,

$$
\mathbf{u}=\boldsymbol{\nabla}\left[-\frac{R^{2} \dot{R}}{r}-U r\left(1+\frac{R^{3}}{2 r^{3}}\right) \cos \theta\right],
$$

where $\theta$ is the polar angle measured from the front stagnation point and $r$ is the distance from the bubble center. In this paper we will mostly take the velocity of the bubble to be a constant.

For the solution of the energy equation (4) we use the same method of Hao and Prosperetti (2000): the temperature field is expanded in a series of Legendre polynomials

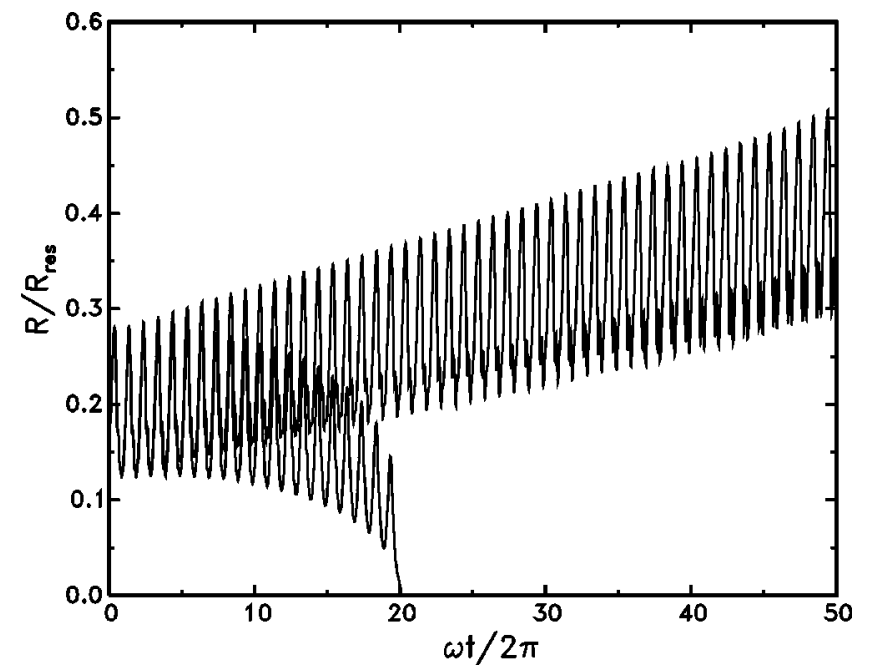

FIG. 1. Nondimensional radius versus time of bubbles translating with constant velocity in water at $95^{\circ} \mathrm{C}$ and $101.3 \mathrm{kPa}$ static pressure in a $1 \mathrm{kHz}$, $30.39 \mathrm{kPa}$ sound field. The upper line is for a velocity of $0.2 \mathrm{~m} / \mathrm{s}$, and the lower line for $0.3 \mathrm{~m} / \mathrm{s}$. The radius is normalized by the resonant radius of a stationary bubble equal to $2.367 \mathrm{~mm}$; the initial radius is $0.5 \mathrm{~mm}$. The initial phase of the sound field promotes expansion.

$$
T=T_{\infty}+\sum_{N=0}^{\infty} S_{N}(r, t) P_{N}(\cos \theta),
$$

and the coupled equations for the various $S_{N}$ that arise upon substitution into (4) are solved by expanding each $S_{N}$ in a series of Chebyshev polynomials; the resulting equations are solved by collocation. The reader is referred to the reference for details.

In the numerical implementation of this scheme we typically use 8 terms in the Legendre expansion (7) and 16 terms in the Chebyshev expansion for the $S_{N}$ 's with 16 collocation points. These values have been chosen on the basis of standard convergence tests. The resulting ordinary differential equation systems are solved by the backward differentiation algorithm of the LSODE package which automatically selects the time step.

In the limit cases of no sound or no translational velocities, we have verified that the code developed for this work gave the same results as those used in our earlier papers dealing with these limit cases (Hao and Prosperetti, 1999, 2000). Those codes had been validated against analytical solutions and independent numerical solutions.

\section{NUMERICAL RESULTS}

The role of convection in the different stages of the process under study is quite different and, accordingly, we treat separately phenomena taking place during the first few acoustic cycles and over a longer time period.

\section{A. Short-time behavior}

Figure 1 compares the evolution of two bubbles, initially both with a radius $R(0)=0.5 \mathrm{~mm}$, subject to a sound field with acoustic amplitude $P_{A}=30.4 \mathrm{kPa}\left(P_{A} / P_{\infty}=0.3\right)$ and frequency $\omega / 2 \pi=1 \mathrm{kHz}$; the liquid is water at $T_{\infty}=95^{\circ} \mathrm{C}$ 


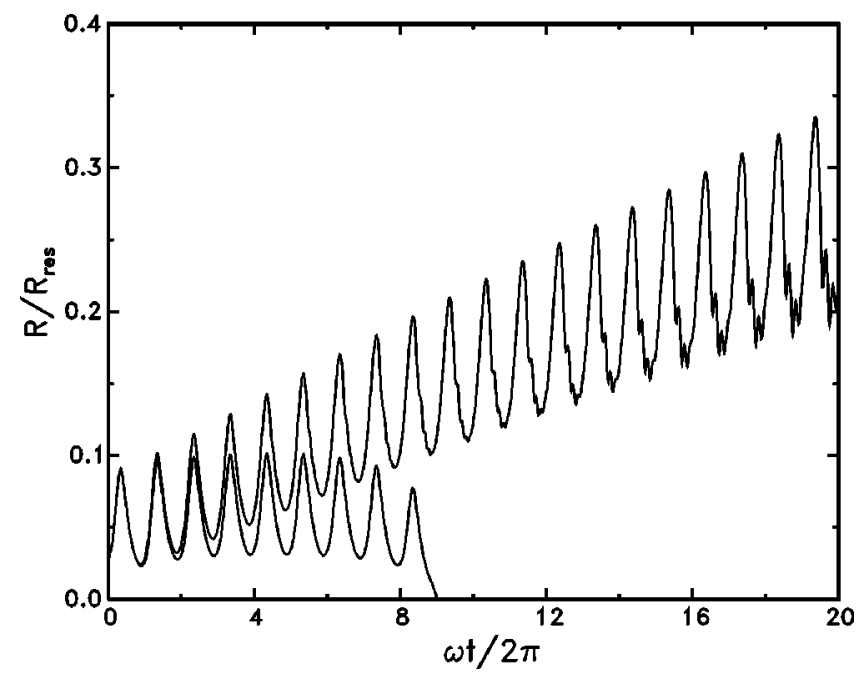

FIG. 2. As in Fig. 1 with $P_{A}=40.52 \mathrm{kPa}$ and $\omega / 2 \pi=0.2 \mathrm{kHz}$; upper line $U=0.2 \mathrm{~m} / \mathrm{s}$, lower line $0.24 \mathrm{~m} / \mathrm{s}$; here $R_{\text {res }}=13.47 \mathrm{~mm}$.

and $P_{\infty}=101.3 \mathrm{kPa}$, so that the subcooling $\Delta T=T_{\text {sat }}\left(P_{\infty}\right)$ $-T_{\infty}$ (in which $T_{\text {sat }}$ is the saturation temperature at $P_{\infty}$ ) is $5{ }^{\circ} \mathrm{C}$. The Jakob number, defined by

$$
\mathrm{Ja}=\rho c_{p L} \frac{T_{\text {sat }}\left(P_{\infty}\right)-T_{\infty}}{L \rho_{V, \text { sat }}\left(P_{\infty}\right)},
$$

in which $c_{p L}$ is the liquid specific heat and $\rho_{V \text {, sat }}\left(P_{\infty}\right)$ is the saturation vapor density at $P_{\infty}$, is 15.2 approximately. The upper curve is for a bubble translating with a constant velocity of $0.2 \mathrm{~m} / \mathrm{s}$, while the lower curve is for a translation velocity of $0.3 \mathrm{~m} / \mathrm{s} ;{ }^{2}$ the radius is normalized by $R_{\text {res }}$, the linear resonant value for a stationary bubble (Hao and Prosperetti, 1999) which here is $2.367 \mathrm{~mm}$. The initial Péclet number, defined by

$$
\mathrm{Pe}=\frac{2 R(0) U}{D}
$$

has values 1184 and 1777 in the two cases. The first bubble grows by rectified diffusion of heat while the other one executes oscillations around a decreasing mean radius until it eventually condenses completely. For comparison, it may be noted that, without sound, condensation is completed at $t$ $=3.0 \mathrm{~ms}(\omega t / 2 \pi=3.0)$ for $U=0.2 \mathrm{~m} / \mathrm{s}$ and at $t=2.5 \mathrm{~ms}$ $(\omega t / 2 \pi=2.5)$ for $U=0.3 \mathrm{~m} / \mathrm{s}$.

A similar behavior is shown in Fig. 2, where $P_{A}$ $=40.52 \mathrm{kPa}\left(P_{A} / P_{\infty}=0.4\right)$ and $\omega / 2 \pi=0.2 \mathrm{kHz}$; the other parameters have the same value as before and $R_{\text {res }}$ $=13.47 \mathrm{~mm}$. At this lower frequency the duration of the compression phase, during which the bubble loses mass, is longer, and a velocity of $0.24 \mathrm{~m} / \mathrm{s}$ (lower line, $\mathrm{Pe}=1,420$ ) is sufficient to cause a complete condensation after a few cycles while for $U=0.2 \mathrm{~m} / \mathrm{s}$ the bubble grows. The behavior shown in these figures is typical of the phenomena encountered during the initial stages of the process object of this study. However, a bubble that survives the first few cycles may actually grow faster when translating. We will address this point shortly. For the time being, we limit our analysis to the initial stages as in Figs. 1 and 2.

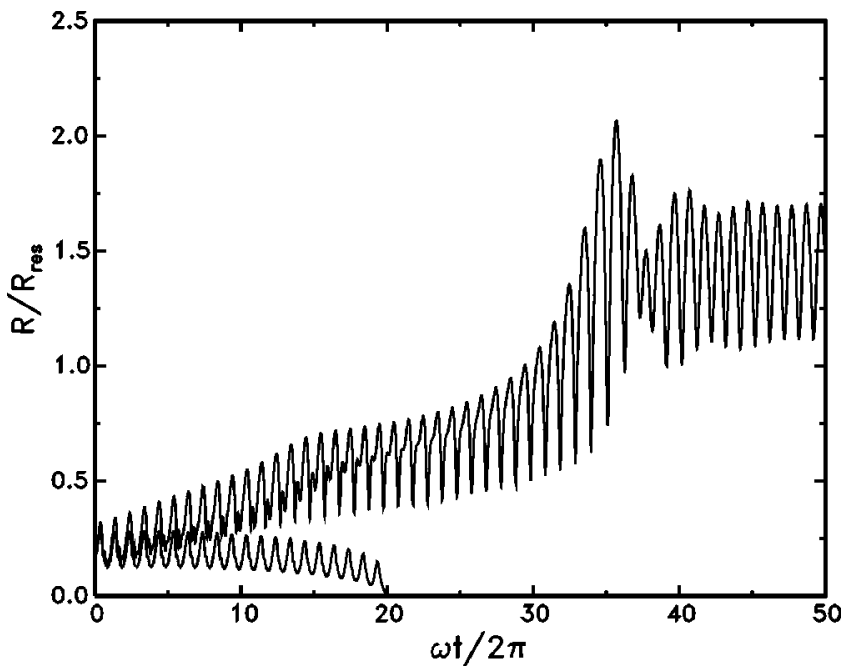

FIG. 3. Nondimensional radius versus time of bubbles translating at $0.3 \mathrm{~m} / \mathrm{s}$ in water at $95^{\circ} \mathrm{C}$ and $101.3 \mathrm{kPa}$ static pressure in a $1 \mathrm{kHz}$, sound field. The upper line is for an acoustic pressure amplitude $P_{A}=35.46 \mathrm{kPa}$, the lower one for $P_{A}=30.39 \mathrm{kPa}$. The radius is normalized by the linear resonant radius, $R_{\text {res }}=2.367 \mathrm{~mm}$, and the initial radius is $0.5 \mathrm{~mm}$. The initial phase of the sound field promotes expansion.

Similar results are found in dependence of other parameters as well. The effect of different acoustic pressures with a fixed velocity, $U=0.3 \mathrm{~m} / \mathrm{s}(\mathrm{Pe}=1,777)$ are shown in Figs. 3 and 4. At $1 \mathrm{kHz}$ (Fig. 3), with acoustic pressure amplitudes $P_{A}=35.46 \mathrm{kPa}$ (upper curve), rectified heat transfer is sufficiently strong to prevent the bubble from collapsing, but if $P_{A}$ is reduced to $30.39 \mathrm{kPa}$, the bubble ultimately condenses. At $0.2 \mathrm{kHz}$ (Fig. 4), the bubble is more labile and one needs a higher pressure amplitude, $P_{A}=45.57 \mathrm{kPa}$ (upper curve), to stabilize it. The effect of frequency is explored directly in Fig. 5, for the same initial radius and subcooling, $P_{A}$ $=40.52 \mathrm{kPa}, U=0.24 \mathrm{~m} / \mathrm{s}$, and acoustic frequencies of 1 $\mathrm{kHz}$ (upper curve) and $0.2 \mathrm{kHz}$. In all these cases the minus sign (initial expansion) is taken in (2).

The general behavior is similar also for smaller bubbles; Figs. 6 and 7 are analogous to Figs. 1 and 4 for $R(0)$ $=0.1 \mathrm{~mm}$. In Fig. $6(1 \mathrm{kHz})$ the acoustic pressure amplitude

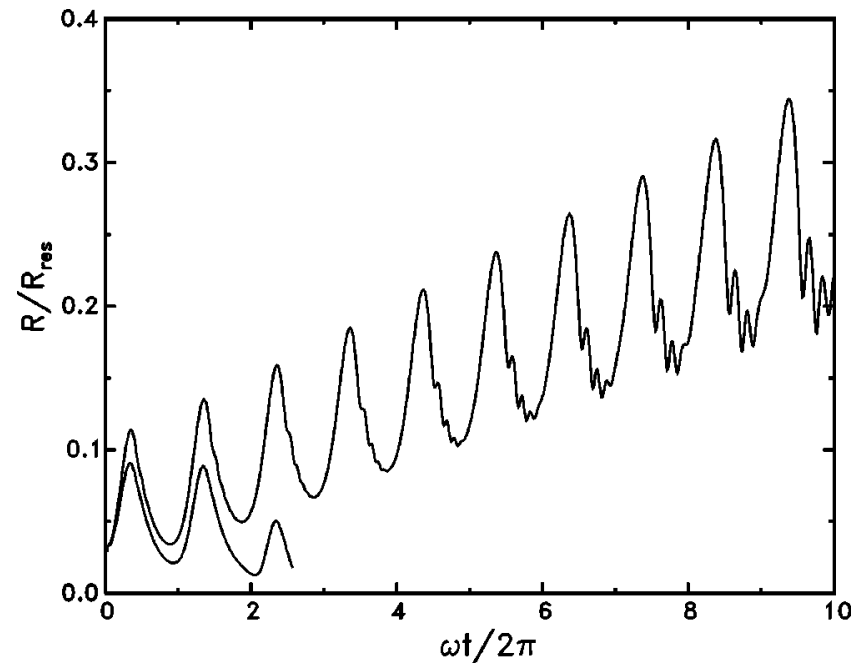

FIG. 4. As in Fig. 3 with $\omega / 2 \pi=0.2 \mathrm{kHz}\left(R_{\text {res }}=13.47 \mathrm{~mm}\right)$; upper curve $P_{A}=45.57 \mathrm{kPa}$; lower curve $P_{A}=40.52 \mathrm{kPa}$. 


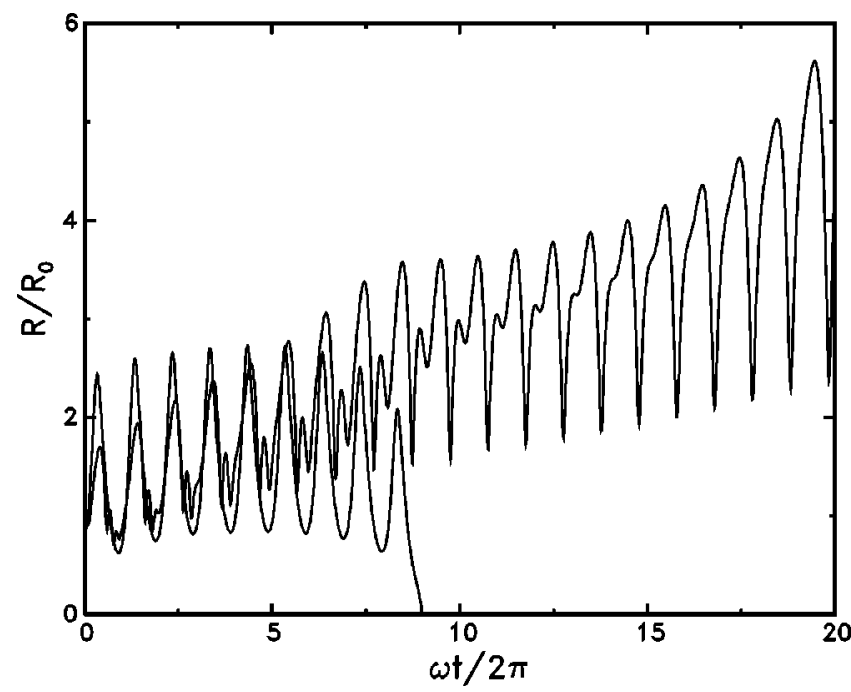

FIG. 5. Normalized radius versus time of bubbles translating at $0.24 \mathrm{~m} / \mathrm{s}$ in water at $95{ }^{\circ} \mathrm{C}$ and $101.3 \mathrm{kPa}$ static pressure in a $40.52 \mathrm{kPa}$ sound field. The upper line is for $\omega / 2 \pi=1 \mathrm{kHz}\left(R_{\text {res }}=2.367 \mathrm{~mm}\right)$, the lower line for $\omega / 2 \pi$ $=0.2 \mathrm{kHz}\left(R_{\mathrm{res}}=13.47 \mathrm{~mm}\right)$. Since the resonant radius depends on frequency, unlike the other figures, here the radius is normalized by the initial value, $R(0)=0.5 \mathrm{~mm}$. The initial phase of the sound field promotes expansion.

is $40.52 \mathrm{kPa}$ and, therefore, the bubble can withstand a bigger velocity $(0.35 \mathrm{~m} / \mathrm{s}$, upper line $)$ without condensing. When the velocity is raised to $0.42 \mathrm{~m} / \mathrm{s}$, however, rectified growth is impossible. An example at a lower frequency, $\omega / 2 \pi=0.2 \mathrm{kHz}$, for two pressure amplitudes $(50.65 \mathrm{kPa}$, upper curve, and $44.57 \mathrm{kPa}$ ) is shown in Fig. 7: upon comparison with the bigger bubble of Fig. 4, it is seen that a stronger forcing is needed to stabilize the bubble. Both Figs. 6 and 7 display the prominent presence of harmonics of the driving sound. These are a nonlinear effect and set in when the growing radius makes the bubble resonant with harmonics of the sound frequency (Hao and Prosperetti, 1999).

These results are affected by the initial temperature distribution around the bubble. As an example, in Fig. 8 we consider the collapsing bubble of Fig. 1 for $U=0.3 \mathrm{~m} / \mathrm{s}$ in an

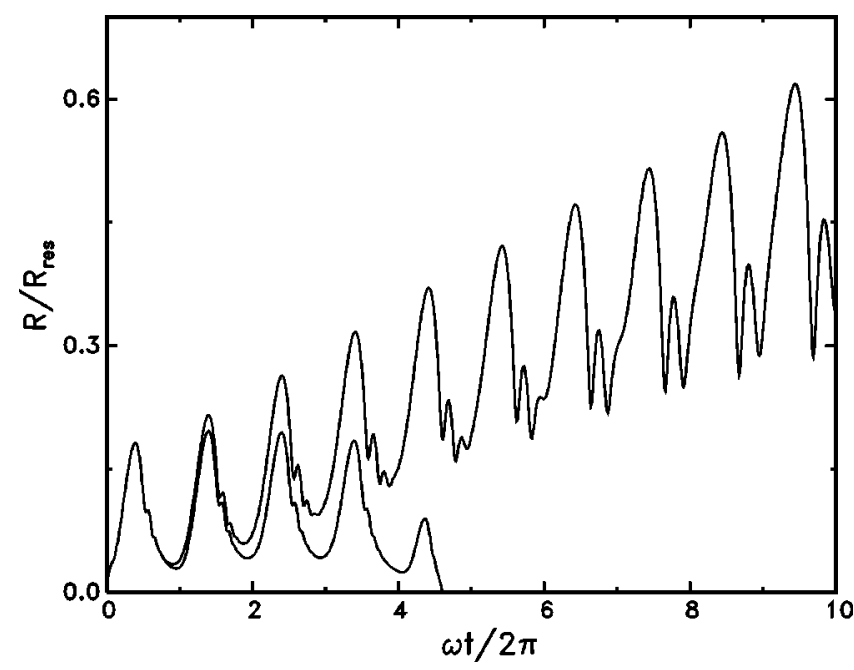

FIG. 6. As in Fig. 1 for an initial radius $R(0)=0.1 \mathrm{~mm}$. The acoustic pressure amplitude is $40.52 \mathrm{kPa}$; upper line, $U=0.35 \mathrm{~m} / \mathrm{s}$, lower line $0.42 \mathrm{~m} / \mathrm{s}$.

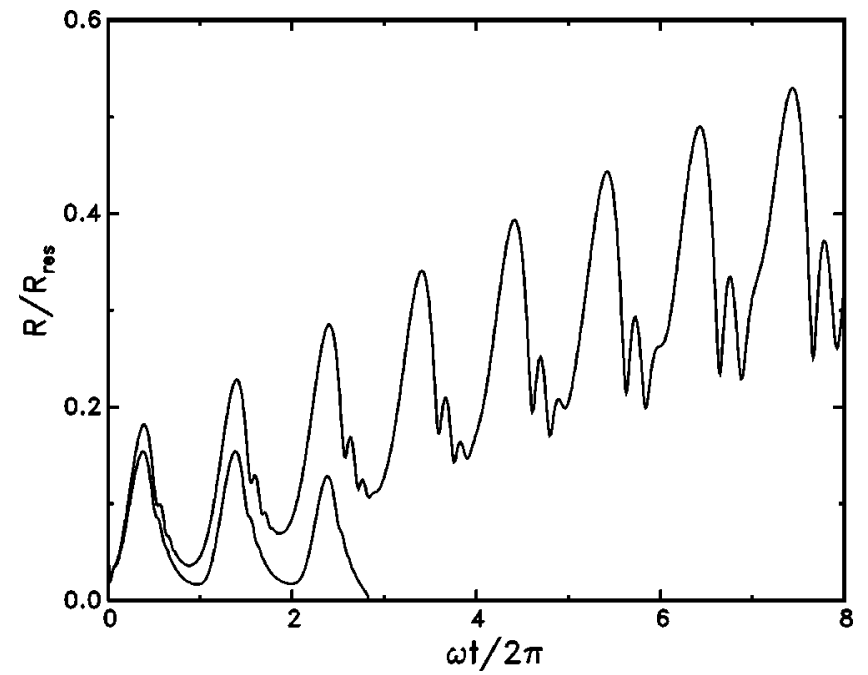

FIG. 7. As in Fig. 4 for an initial radius $R(0)=0.1 \mathrm{~mm} ; U=0.3 \mathrm{~m} / \mathrm{s}$; upper line $P_{A}=50.65 \mathrm{kPa}$, lower line $44.57 \mathrm{kPa}$.

initial temperature field given, for $R \leqslant r \leqslant R+\delta$, by

$$
T(r, \theta, 0)=T_{\infty}+\left[T_{\text {sat }}\left(P_{\infty}\right)-T_{\infty}\right]\left(1-\frac{r-R(0)}{\delta}\right)^{2},
$$

with $\delta / R(0)=0.5$ (solid line). The dashed line is the same result plotted in Fig. 1, with $T(r, \theta, 0)=T_{\infty}$. With the initial temperature distribution (10) the bubble grows. As $\delta / R(0)$ is reduced, the growth process is found to slow down.

These results illustrate the sensitivity of the boundary between stable and unstable bubbles to the precise initial temperature distribution in the bubble neighborhood. ${ }^{3}$ In this regard, the situation is similar to that encountered in Hao and Prosperetti (2000) for bubbles in the absence of sound. In view of the marked dependence of the phenomenon on such details and of the abundance of parameters and physical

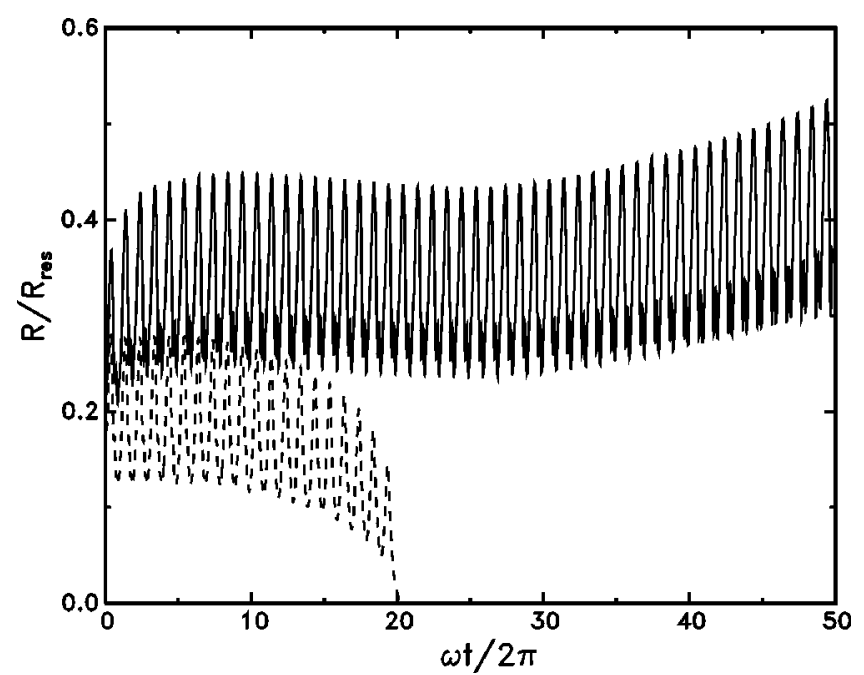

FIG. 8. Nondimensional radius versus time of bubbles translating with constant velocity $U=0.3 \mathrm{~m} / \mathrm{s}$ in water at $95^{\circ} \mathrm{C}$ and $101.3 \mathrm{kPa}$ static pressure in a $1 \mathrm{kHz}, 30.39 \mathrm{kPa}\left(P_{A} / P_{\infty}=0.3\right)$ sound field for two initial temperature distributions. For the dashed line $T(r, \theta, 0)=T_{\infty}$, while, for the solid line, the initial temperature distribution is given by (10) with $\delta / R(0)=0.5$. The radius is normalized by the resonant radius of a stationary bubble equal to $2.367 \mathrm{~mm}$; the initial radius is $0.5 \mathrm{~mm}$. The initial phase of the sound field promotes expansion. 


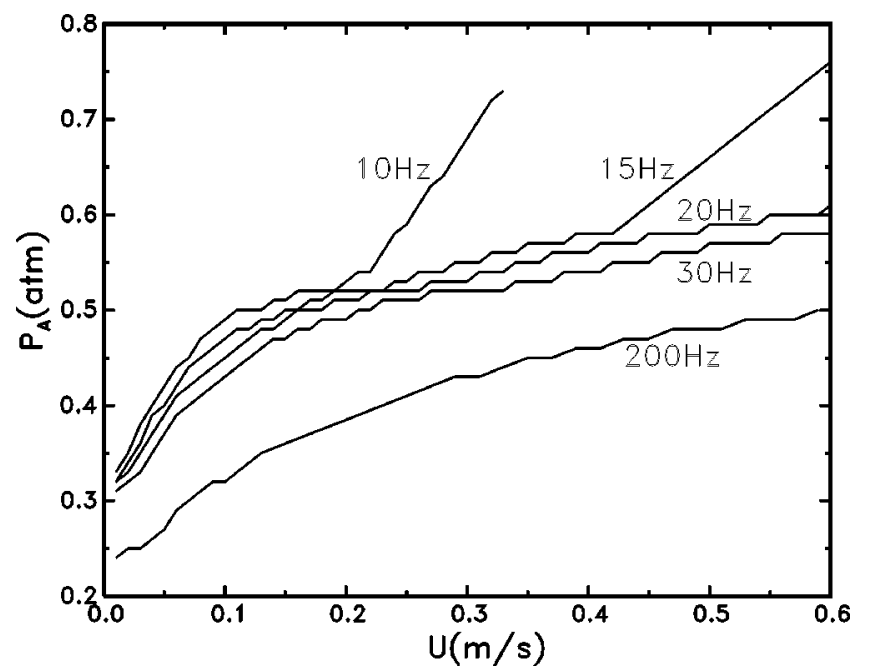

FIG. 9. Approximate location of the stability boundary for a vapor bubble translating with velocity $U$ in a sound field with pressure amplitude $P_{A}$ for different values of the sound frequency; the initial bubble radius is $0.5 \mathrm{~mm}$. The liquid is water at $T_{\infty}=95^{\circ} \mathrm{C}$ and $P_{\infty}=101.3 \mathrm{kPa}$ and, at $t=0$, $T=T_{\infty}$, everywhere. The bubble grows by rectified diffusion for acoustic amplitudes above the lines. The initial phase of the sound is expansive [minus sign in Eq. (2)]. The curves are labeled by the sound frequency in $\mathrm{Hz}$.

properties, it is somewhat futile to attempt a complete quantitative characterization of the stability limit. Thus, we only present a rather crude characterization of parameter space in Figs. $9[R(0)=0.5 \mathrm{~mm}]$ and $10[R(0)=0.1 \mathrm{~mm}]$ for an initial temperature equal to $T_{\infty}=95^{\circ} \mathrm{C}$ everywhere and $P_{\infty}$ $=101.3 \mathrm{kPa}$. The figures show approximate stability lines in the $\left(P_{A}, U\right)$ plane for different sound frequencies: for acoustic amplitudes above these lines the bubble grows by rectified diffusion of heat, while it eventually collapses for smaller $P_{A}$ 's.

These results have been obtained with an initially expansive phase of the sound field [minus sign in Eq. (2)], as the bubble behavior is strongly affected by this phase. A typical near-threshold behavior with an expansive initial phase [minus sign in Eq. (2)] is shown in Fig. 11, where $R(0)$

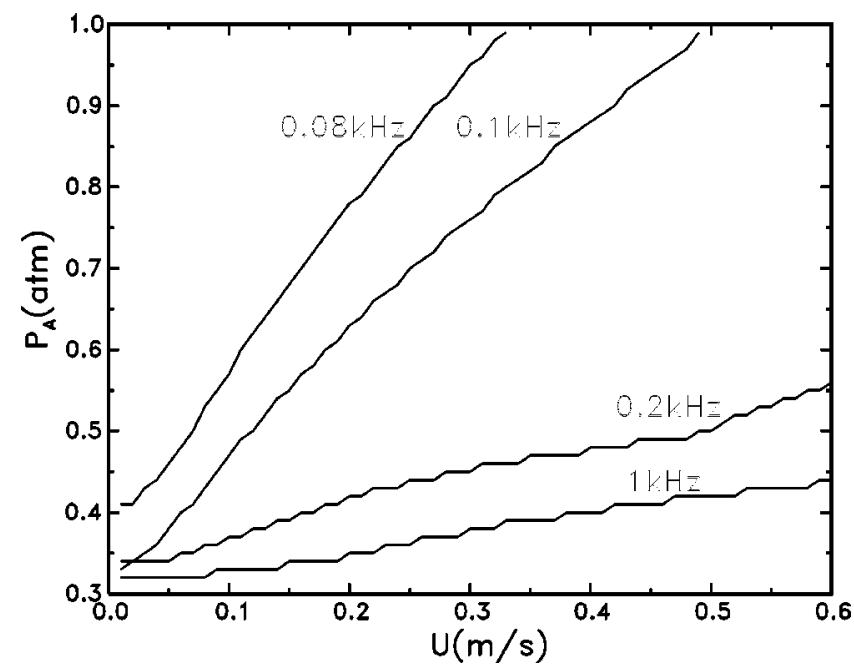

FIG. 10. As in Fig. 9 for $R(0)=0.1 \mathrm{~mm}$. The curves are labeled by the sound frequency in $\mathrm{kHz}$.

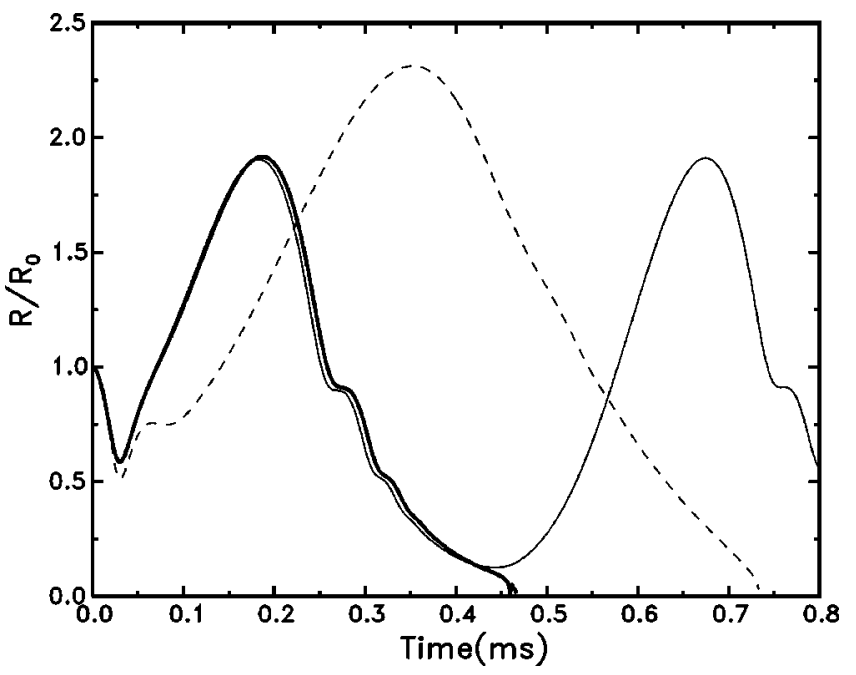

FIG. 11. Examples of the initial bubble behavior with an expansive sound phase [minus sign in Eq. (2)] with $R(0)=0.1 \mathrm{~mm}, P_{A}=30.39 \mathrm{kPa}, U$ $=0.2 \mathrm{~m} / \mathrm{s}$ and $T_{\text {sat }}-T_{\infty}=5{ }^{\circ} \mathrm{C} ; 1 \mathrm{kHz}$ (dashed line), $2 \mathrm{kHz}$ (thick solid line), $2.05 \mathrm{kHz}$. The threshold for growth lies between 2 and $2.05 \mathrm{kHz}$.

$=0.1 \mathrm{~mm}, \quad P_{A}=30.39 \mathrm{kPa}, \quad U=0.2 \mathrm{~m} / \mathrm{s} \quad$ and $\quad T_{\text {sat }}-T_{\infty}$ $=5{ }^{\circ} \mathrm{C}$. Both for $1 \mathrm{kHz}$ (dashed line) and $2 \mathrm{kHz}$ (thick solid line) the bubble condenses completely after a first expansion but, for $2.05 \mathrm{kHz}$, there is sufficient rectified heat transfer to stabilize the bubble and actually promote growth (the latter not shown in the figure). Figure 12 is for an initial compression phase [plus sign in Eq. (2)]: in the absence of sound (dash-and-dot line) or at $100 \mathrm{~Hz}$ (short dashes) a relatively slow condensation takes place; at the higher frequencies of 1 $\mathrm{kHz}$ (long dashes) and $3.8 \mathrm{kHz}$ (thick solid line) condensation is more rapid, while at $3.9 \mathrm{kHz}$ a strong growth is encountered.

It is interesting to briefly examine the consequences of the assumption of spatially uniform vapor conditions inside the bubble. In order to study this issue precisely, it would be necessary to account for the vapor flow induced by the translation of the bubble, which is a nontrivial task. Thus, we

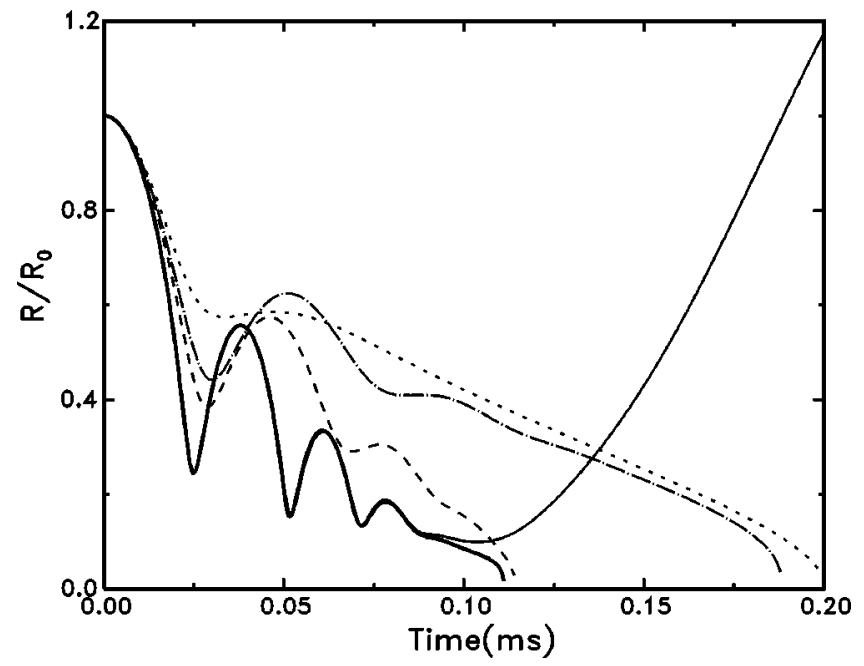

FIG. 12. Examples of the initial bubble behavior with a compressive sound phase [plus sign in Eq. (2)] with $R(0)=0.1 \mathrm{~mm}, P_{A}=30.39 \mathrm{kPa}, U$ $=0.2 \mathrm{~m} / \mathrm{s}$ and $T_{\text {sat }}-T_{\infty}=5{ }^{\circ} \mathrm{C}$; no sound (dash-and-dot line), $100 \mathrm{~Hz}$ (short dashes), $1 \mathrm{kHz}$ (long dashes), $3.8 \mathrm{kHz}$ (thick solid line), $3.9 \mathrm{kHz}$. 


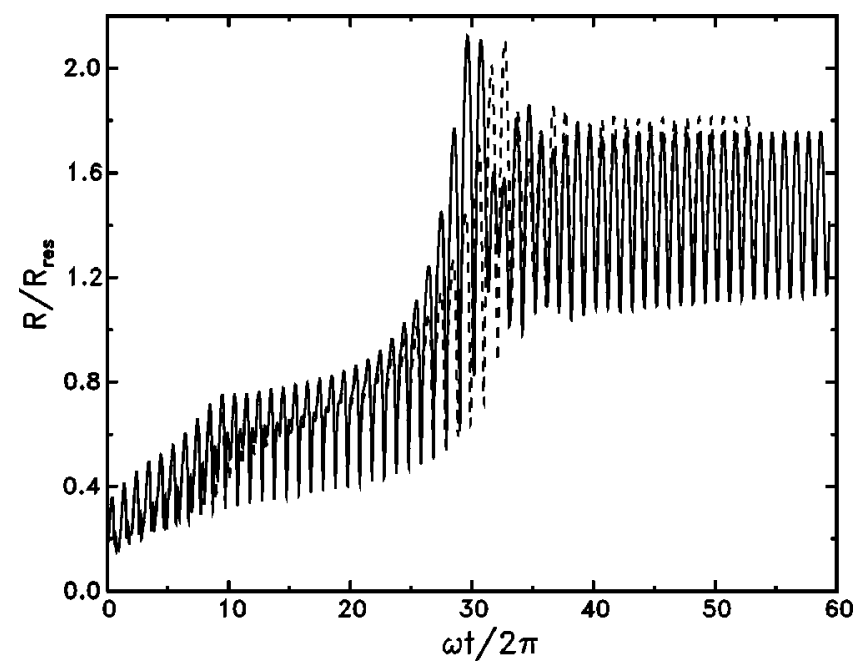

FIG. 13. Comparison between the growth rate of a stationary bubble modeled with a uniform (solid line) and nonuniform interior. The conditions are $R(0)=0.5 \mathrm{~mm}, P_{A}=40.9 \mathrm{kPa}\left(P_{A} / P_{\infty}=0.4\right), \omega / 2 \pi=1 \mathrm{kHz}, T_{\infty}=95^{\circ} \mathrm{C}$ and $P_{\infty}=101.3 \mathrm{kPa}$.

content ourselves with considering the difference between homogeneous and nonhomogeneous conditions for a stationary bubble. Figure 13 shows a typical result. The conditions are: $R(0)=0.5 \mathrm{~mm}, P_{A}=40.9 \mathrm{kPa}\left(P_{A} / P_{\infty}=0.4\right), \omega / 2 \pi$ $=1 \mathrm{kHz}, T_{\infty}=95^{\circ} \mathrm{C}$ and $P_{\infty}=101.3 \mathrm{kPa}$. The solid line is for spatially uniform vapor conditions, while the dashed line accounts for vapor nonuniformity according to our earlier model for a stationary bubble (Hao and Prosperetti, 1999). It is seen here that spatial nonuniformity gives rise to a somewhat slower growth. This result is not surprising: there is a tendency to develop stronger vapor temperature gradients near the interface during condensation than evaporation, because the departing vapor tends to "wash them out" [for explicit results on the temperature distribution inside the bubble, see Prosperetti and Hao (2002)]. Now, during condensation, the bubble surface temperature rises and, therefore, if the vapor temperature is allowed to be spatially nonuniform, there will be conduction of heat into the vapor, which favors condensation and hence heat loss. In any case, relatively large differences are only found near the resonance radius, where the oscillation amplitude is strongest. The vapor circulation neglected in this result would have the effect of mixing the vapor and may be expected, therefore, to decrease the consequences of spatial nonuniformity.

\section{B. Long-time behavior}

It would be wrong to conclude from the results shown so far that translation in a subcooled liquid always hinders bubble growth. An example is shown in Fig. 14, where the conditions are as in the previous figure. The solid line is for a stationary bubble, while the dashed line is for a bubble translating at $0.3 \mathrm{~m} / \mathrm{s}$. Convection increases the heat loss during compression as well as the heat gain during expansion: this result therefore implies that the latter effect is greater than the former. That this must be so can be understood from the approximate expression derived by Rucken-

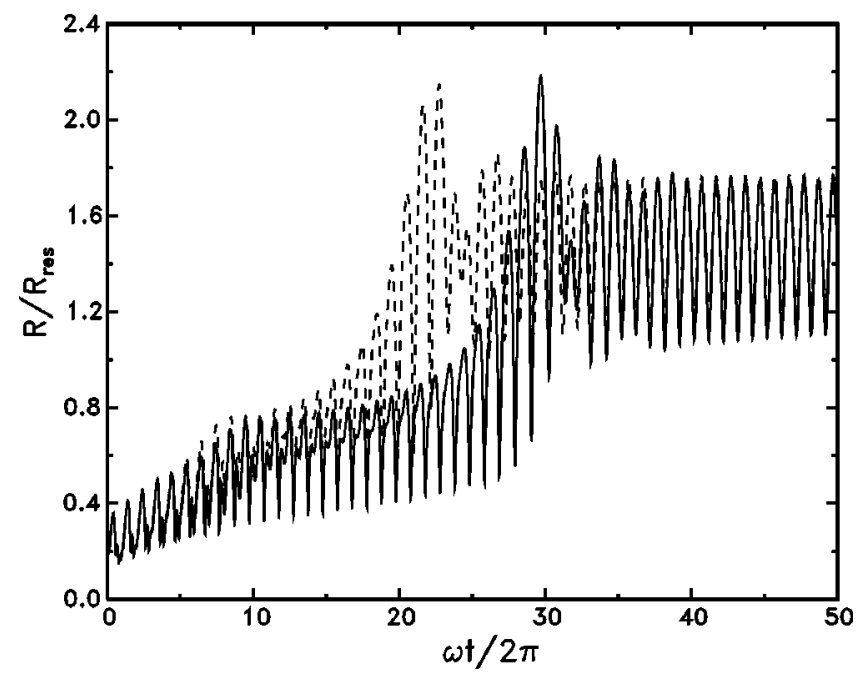

FIG. 14. Comparison between the growth rate of a stationary (solid line) and translating bubble. The conditions are $R(0)=0.5 \mathrm{~mm}, U=0.3 \mathrm{~m} / \mathrm{s}$, $P_{A}=40.9 \mathrm{kPa} \quad\left(P_{A} / P_{\infty}=0.4\right), \quad \omega / 2 \pi=1 \mathrm{kHz}, \quad T_{\infty}=95^{\circ} \mathrm{C} \quad$ and $P_{\infty}$ $=101.3 \mathrm{kPa}$.

stein (1959) for the Nusselt number for flow past a sphere of constant diameter in the limit of large Péclet numbers (see also Legendre et al., 1998):

$$
\mathrm{Nu}=\frac{2 R h}{k}=2 \sqrt{\frac{P e}{\pi}},
$$

in which $h$ is the heat transfer coefficient. The instantaneous heat transfer is proportional to $h R^{2}$, i.e., for constant $U$ and temperature difference, to $R^{-1 / 2} R^{2}=R^{3 / 2}$, i.e., greater during expansion.

In all the cases considered so far the bubble velocity was held fixed. In reality, a translating and pulsating bubble will have a variable velocity due to changes in added mass, drag, and possibly buoyancy. Details of the velocity dependence on bubble volume depend in a subtle way on Reynolds and Weber numbers. A simple model, which we used in an earlier paper (Hao and Prosperetti, 2000), consists in assuming that the bubble remains spherical and the impulse is conserved:

$$
\frac{2}{3} \pi \rho R^{3} U=\frac{2}{3} \pi \rho R(0)^{3} U(0) .
$$

Figure 15 compares a constant velocity case, $U=0.3 \mathrm{~m} / \mathrm{s}$ (dashed line), with a situation in which $U(0)=0.3 \mathrm{~m} / \mathrm{s}$, but $U$ at later times is found from Eq. (12). The pressure amplitude is $P_{A}=40.9 \mathrm{kPa}\left(P_{A} / P_{\infty}=0.4\right), \omega / 2 \pi=1 \mathrm{kHz}, T_{\infty}$ $=95^{\circ} \mathrm{C}, P_{\infty}=101.3 \mathrm{kPa}$, and $R(0)=0.5 \mathrm{~mm}$. The figure shows that allowing the velocity to vary slows down the growth of the bubble. This result can be understood by noting that, according to (12), the translational velocity increases when the bubble shrinks, which increases heat loss, while it decreases when the bubble expands, which is the phase of the acoustic cycle during which heat is gained.

In a saturated or superheated liquid, the growth of a bubble is always enhanced by motion relative to the liquid. An example is shown in Fig. 16, where the water is saturated at $101.3 \mathrm{kPa}$, the acoustic pressure amplitude is $30.39 \mathrm{kPa}$, the frequency is $1 \mathrm{kHz}$, and the initial bubble radius is 0.5 $\mathrm{mm}$. The solid line is for a bubble translating at $0.3 \mathrm{~m} / \mathrm{s}$, 


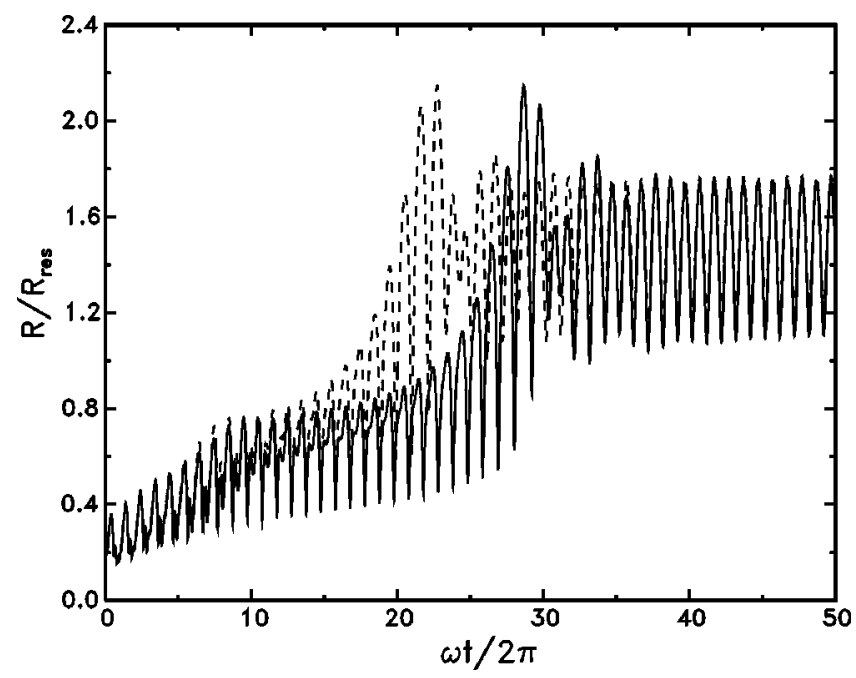

FIG. 15. Comparison between the growth rate of a bubble translating with fixed velocity $U=0.3 \mathrm{~m} / \mathrm{s}$ (dashed line), and a variable velocity calculated from (12). The conditions are $R(0)=0.5 \mathrm{~mm}, P_{A}=40.9 \mathrm{kPa} \quad\left(P_{A} / P_{\infty}\right.$ $=0.4), \omega / 2 \pi=1 \mathrm{kHz}, T_{\infty}=95^{\circ} \mathrm{C}$ and $P_{\infty}=101.3 \mathrm{kPa}$.

while the dashed line is for a stationary bubble; the radius is normalized by the resonant radius of a stationary bubble equal to $2.71 \mathrm{~mm}$.

\section{DISCUSSION}

The numerical results of the previous section, as well as the theory of rectified heat transfer with no translation (see, e.g., Gumerov, 2000), illustrate the complexity of the phenomenon under study. It is not possible to give a simple yet detailed physical interpretation of the results presented in the previous section: we shall content ourselves with some rather general arguments.

The most dangerous phase of the process for the survival of the bubble is the initial one and, accordingly, we limit ourselves to this stage. It may be noted, however, that, if the bubble velocity is large enough to move by about one diam-

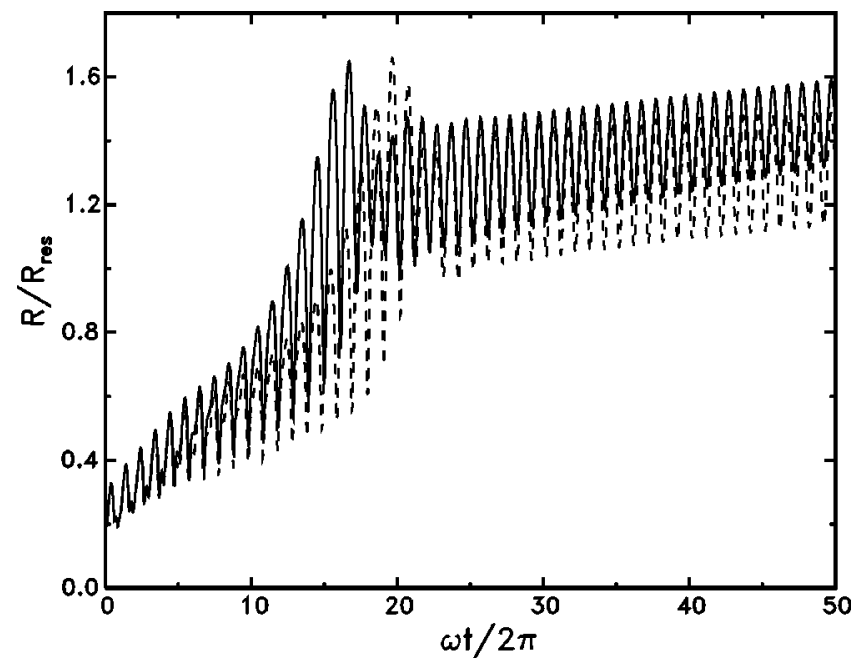

FIG. 16. Nondimensional radius versus time of a bubble translating at 0.3 $\mathrm{m} / \mathrm{s}$ in water at $100{ }^{\circ} \mathrm{C}$ and $101.3 \mathrm{kPa}$ in a $1 \mathrm{kHz}, 30.39 \mathrm{kPa}$ sound field (solid line); the dashed line is for a stationary bubble. The radius is normalized by the resonant radius of a stationary bubble equal to $2.71 \mathrm{~mm}$; the initial radius is $0.5 \mathrm{~mm}$. eter or more during one cycle of the sound, the surrounding temperature field will approximately always be close to the initial undisturbed distribution and, therefore, the bubble behavior during the initial stage may be expected to characterize also its long-term behavior. A bubble translating with a velocity $U$ will move a distance equal to its diameter after a number $N$ of acoustic cycles given by

$$
N=\frac{\omega}{2 \pi} \frac{2 R}{U} .
$$

For $R=0.5 \mathrm{~mm}, \omega / 2 \pi=1 \mathrm{kHz}, N=1$ when $U$ has the rather large value of $1 \mathrm{~m} / \mathrm{s}$. These conditions, therefore, are probably not frequently encountered.

In the first place, it is obvious that no rectified heat transfer is possible unless the liquid becomes temporarily superheated during the expansion phase of the sound. With the aid of the Clausius-Clapeyron relation, this condition gives

$$
P_{A} \geqslant L \rho_{V} \frac{T_{\text {sat }}-T_{\infty}}{T_{\text {sat }}} .
$$

For water at $T_{\infty}=95^{\circ} \mathrm{C}$ and $P_{\infty}=101.3 \mathrm{kPa}$, we find $P_{A}$ $\geqslant 18.2 \mathrm{kPa}$. When this relation is barely satisfied, there is only a small fraction of the sound cycle during which the liquid is momentarily superheated. Clearly, if this interval of time is too short, the energy gain will not be sufficient to compensate the loss during the remainder of the cycle. Hence, one may expect that the estimate (14) will actually be lower than the true threshold; this conclusion is in agreement with the behavior shown in the previous figures.

Another condition for stability is that the bubble should not condense completely during the compression half-cycle of the sound, before the expansion phase renders the liquid temporarily superheated. To estimate this low-frequency limit, we may use a simplified form of the heat balance at the bubble surface:

$$
q=\rho_{V} L \dot{R}
$$

where $q$ is the liquid-side heat flux. This relation is based on the assumption that the vapor-side heat flux is negligible and

TABLE I. Bubble collapse time for stationary vapor bubbles in water at $95^{\circ} \mathrm{C}$ and $101.3 \mathrm{kPa}$ as calculated numerically and as estimated from Eq. (17).

\begin{tabular}{cccc}
\hline \hline & $\begin{array}{c}\text { Collapse time }(\mathrm{ms}) \\
R_{0}(\mu \mathrm{m})\end{array}$ & $\begin{array}{c}\text { Collapse time }(\mathrm{ms}) \\
\text { (Numerical) }\end{array}$ & Ratio \\
\hline 50 & 0.0788 & 0.052 & 1.51 \\
60 & 0.09 & 0.075 & 1.2 \\
70 & 0.112 & 0.102 & 1.1 \\
80 & 0.141 & 0.133 & 1.06 \\
90 & 0.178 & 0.168 & 1.06 \\
100 & 0.22 & 0.21 & 1.05 \\
200 & 1.06 & 0.83 & 1.28 \\
300 & 2.69 & 1.87 & 1.44 \\
400 & 5.14 & 3.32 & 1.55 \\
500 & 8.5 & 5.18 & 1.64 \\
600 & 12.8 & 7.47 & 1.72 \\
700 & 17.8 & 10.2 & 1.75 \\
800 & 23.4 & 13.3 & 1.76 \\
\hline \hline
\end{tabular}


TABLE II. Comparison of the approximate expression (18) for the frequency above which a bubble would survive the first compression phase of the sound field, evaluated both in terms of $\mathrm{Ja}$ [Eq. (8)] and $\mathrm{Ja}_{e}$ [Eq. (19)], with numerical results obtained with an initially compressive phase of the sound field [plus sign in Eq. (2)]. The liquid is water at $95^{\circ} \mathrm{C}$ and $101.3 \mathrm{kPa}$ and the bubbles are stationary.

\begin{tabular}{cccccc}
\hline \hline $\begin{array}{c}R_{0} \\
(\mathrm{~mm})\end{array}$ & $\begin{array}{c}P_{A} \\
(\mathrm{kPa})\end{array}$ & $\begin{array}{c}\text { Numerical } \\
(\text { First cycle })\end{array}$ & $\begin{array}{c}\text { Numerical } \\
(\text { Growth })\end{array}$ & $\begin{array}{c}\text { Eq. (18) } \\
\text { with Ja }\end{array}$ & $\begin{array}{c}\text { Eq. } \\
\text { (18) } \\
\text { with Ja }\end{array}$ \\
\hline 0.5 & 20.26 & $100 \pm 10 \mathrm{~Hz}$ & $3.7 \pm 0.1 \mathrm{kHz}$ & $98.8 \mathrm{~Hz}$ & $288 \mathrm{~Hz}$ \\
0.5 & 22.29 & $101 \pm 1 \mathrm{~Hz}$ & $125 \pm 5 \mathrm{~Hz}$ & $98.8 \mathrm{~Hz}$ & $306 \mathrm{~Hz}$ \\
0.5 & 30.39 & $130 \pm 10 \mathrm{~Hz}$ & $130 \pm 10 \mathrm{~Hz}$ & $98.8 \mathrm{~Hz}$ & $374 \mathrm{~Hz}$ \\
0.1 & 20.26 & $3.2 \pm 0.1 \mathrm{kHz}$ & $17.5 \pm 0.5 \mathrm{kHz}$ & $2.47 \mathrm{kHz}$ & $7.2 \mathrm{kHz}$ \\
0.1 & 22.29 & $3.3 \pm 0.1 \mathrm{kHz}$ & $4.2 \pm 0.1 \mathrm{kHz}$ & $2.47 \mathrm{kHz}$ & $7.8 \mathrm{kHz}$ \\
0.1 & 30.39 & $3.6 \pm 0.1 \mathrm{kHz}$ & $3.6 \pm 0.1 \mathrm{kHz}$ & $2.47 \mathrm{kHz}$ & $9.3 \mathrm{kHz}$ \\
\hline \hline
\end{tabular}

that all the heat conducted into the liquid accounts for the latent heat released by the saturated vapor condensing at the bubble surface. If, following Florschuetz and Chao (1965), we use for $q$ the estimate $q \simeq k\left(T_{\text {sat }}-T_{\infty}\right) / \sqrt{\pi D t}$, we find

$$
\frac{R(t)}{R(0)}=1-2 \mathrm{Ja} \sqrt{\frac{D t}{\pi R^{2}(0)}},
$$

from which, upon setting $R\left(t_{c}\right)=0$, one deduces the following estimate for the characteristic bubble collapse time $t_{c}$ :

$$
t_{c}=\frac{\pi}{4 \mathrm{Ja}^{2}} \frac{R^{2}(0)}{D} \text {. }
$$

As shown in Table I, while this estimate of the collapse time is of the right order of magnitude, depending on specific conditions, numerically it may be off by as much as a factor of 2 . With this caveat in mind, if we set $t_{c} \geqslant \pi / \omega$ for stability, we find

$$
\frac{\omega}{2 \pi} \geqslant \frac{2 D}{\pi R^{2}(0)} \mathrm{Ja}^{2} .
$$

As the static pressure in the liquid changes due to the presence of the sound field, the instantaneous Jakob number also changes; in particular, at the pressure maximum, it has an "effective" value given by

$$
\mathrm{Ja}_{e}=\rho c_{p L} \frac{T_{\mathrm{sat}}\left(P_{\infty}+P_{A}\right)-T_{\infty}}{L \rho_{V, \mathrm{sat}}\left(P_{\infty}+P_{A}\right)},
$$

where the variation of $L, \rho$, and $c_{p L}$ has been neglected for simplicity.

A comparison of (18), evaluated both in terms of Ja and $\mathrm{Ja}_{e}$, with some numerical results is given in Table II. It should be noted that these results have been obtained with an initially compressive phase of the sound field [i.e., the plus sign in Eq. (2)] because otherwise the correct value of the radius to use would be that at the end of the first expansion, which it is difficult to estimate. It is seen that, in general, the frequency threshold lies somewhere between the two predictions of Eq. (18). This estimate refers to the survival of the bubble during the first sound cycle but, in the table, we also show the numerically determined threshold for a sustained growth of the bubble. The substantial difference between the two numerical thresholds that appears in some cases arises when the acoustic pressure does not exceed by much the very low estimate of Eq. (14).

The previous estimate is applicable at low translational velocities, when the heat loss from the bubble is dominated by conduction. When convection dominates, we may estimate $q$ from the expression (11) of the Nusselt number. Upon setting in (15)

$$
q=\operatorname{Nu} k \frac{T_{\mathrm{sat}}-T_{\infty}}{2 R}=2 k\left(T_{\mathrm{sat}}-T_{\infty}\right) \sqrt{\frac{U}{2 \pi D R(t)}},
$$

and integrating, we find

$$
\frac{R(t)}{R(0)}=\left(1-3 \mathrm{Ja} \sqrt{\frac{D U}{2 \pi R^{3}(0)}} t\right)^{2 / 3},
$$

from which

$$
t_{c}^{\prime}=\frac{1}{3 \mathrm{Ja}} \sqrt{\frac{2 \pi R^{3}(0)}{D U}}=\frac{R^{2}(0)}{3 \mathrm{Ja} D} \sqrt{\frac{2 \pi}{\operatorname{Pe}(0)}} .
$$

\begin{tabular}{|c|c|c|c|c|c|c|}
\hline \multirow[b]{3}{*}{$R_{0}(\mathrm{~mm})$} & \multicolumn{6}{|c|}{ Collapse time (ms) } \\
\hline & \multicolumn{3}{|c|}{ Numerical } & \multicolumn{3}{|c|}{ Eq. (22) } \\
\hline & $0.2 \mathrm{~m} / \mathrm{s}$ & $0.4 \mathrm{~m} / \mathrm{s}$ & $0.6 \mathrm{~m} / \mathrm{s}$ & $0.2 \mathrm{~m} / \mathrm{s}$ & $0.4 \mathrm{~m} / \mathrm{s}$ & $0.6 \mathrm{~m} / \mathrm{s}$ \\
\hline 0.05 & 0.070 & 0.063 & 0.055 & 0.11 & 0.076 & 0.062 \\
\hline 0.075 & 0.10 & 0.09 & 0.08 & 0.20 & 0.14 & 0.11 \\
\hline 0.10 & 0.18 & 0.15 & 0.13 & 0.30 & 0.21 & 0.17 \\
\hline 0.25 & 1.0 & 0.73 & 0.60 & 1.2 & 0.85 & 0.69 \\
\hline 0.50 & 3.0 & 2.2 & 1.8 & 3.4 & 2.4 & 2.0 \\
\hline 0.75 & 5.6 & 4.0 & 3.2 & 6.2 & 4.4 & 3.6 \\
\hline
\end{tabular}

The ratio of the two collapse times is

$$
\frac{t_{c}}{t_{c}^{\prime}}=\frac{3}{8 \mathrm{Ja}} \sqrt{\pi \mathrm{Pe}(0)}
$$

TABLE III. The numerically calculated collapse time of bubbles translating with constant velocity in water at $95{ }^{\circ} \mathrm{C}$ and $101.3 \mathrm{kPa}$ in the absence of a sound field compared with the estimate (22). Note that for smaller bubbles at low velocity the Péclet number is too small to justify use of (22). 
TABLE IV. Comparison of the estimate (25) for the frequency above which a translating bubble would survive the first compression phase of the sound field, with numerical results obtained with an initially compressive phase of the sound field [plus sign in Eq. (2)]. For each bubble radius, the numerical result in the first line is the threshold for the bubble to survive the first compression induced by the sound field; that in the second line is the threshold for bubble growth by rectified heat transfer. The liquid is water at $95^{\circ} \mathrm{C}$ and $101.3 \mathrm{kPa}$ and the acoustic pressure amplitude is $30.39 \mathrm{kPa}$.

Threshold (kHz)

\begin{tabular}{ccccccccc} 
& \multicolumn{3}{c}{ Numerical (First cycle/growth) } & & \multicolumn{2}{c}{ Estimate from Eq. (25), with Ja $/ \mathrm{Ja}$} \\
\cline { 2 - 4 }$R_{0}(\mathrm{~mm})$ & $0.2 \mathrm{~m} / \mathrm{s}$ & $0.4 \mathrm{~m} / \mathrm{s}$ & $0.6 \mathrm{~m} / \mathrm{s}$ & & $0.2 \mathrm{~m} / \mathrm{s}$ & $0.4 \mathrm{~m} / \mathrm{s}$ & $0.6 \mathrm{~m} / \mathrm{s}$ \\
\hline \multirow{2}{*}{0.5} & $0.275 \pm 0.025 /$ & $0.425 \pm 0.025 /$ & $0.525 \pm 0.025 /$ & & $0.290 /$ & $0.410 /$ & $0.502 /$ \\
& $0.700 \pm 0.050$ & $1.45 \pm 0.050$ & $1.85 \pm 0.050$ & & 0.150 & 0.212 & 0.260 \\
\multirow{2}{*}{0.1} & $3.85 \pm 0.05 /$ & $4.45 \pm 0.05 /$ & $5.45 \pm 0.05 /$ & & $3.24 /$ & & $4.58 /$ & $5.60 /$ \\
& $3.85 \pm 0.05$ & $5.35 \pm 0.05$ & $8.5 \pm 0.05$ & & 1.68 & 2.37 & 2.90 \\
\hline \hline
\end{tabular}

and we may therefore conclude that convection dominates for $\mathrm{Pe}(0)$ greater than the critical value

$$
\mathrm{Pe}_{c}=\frac{64}{9 \pi} \mathrm{Ja}^{2} .
$$

The collapse time estimated from (22) is compared with some numerical results in Table III. The Péclet number for $R(0)=0.05 \mathrm{~mm}$ and $U=0.2 \mathrm{~m} / \mathrm{s}$ is 119 , well below the estimate (24) of $\mathrm{Pe}_{c}$ which, for this subcooling, is 523. This case is therefore conduction dominated and, not surprisingly, the estimate based on (22) is rather poor. As Pe increases, however, the table shows that there is a good agreement with the numerical results.

The same argument as before applied to (22) now gives

$$
\frac{\omega}{2 \pi} \geqslant \frac{3 D \mathrm{Ja}}{4 R^{2}(0)} \sqrt{\frac{\mathrm{Pe}(0)}{\pi}} \text {. }
$$

This estimate is compared with some numerical results in Table IV whereas, in Table III, separate thresholds are shown for bubble survival during the first compressive half-cycle of the sound and for bubble growth by rectified heat transfer. The first threshold is estimated rather well by (25). Upon comparing the results of Tables II and IV, it is seen that, for a stationary bubble, the stability threshold is better predicted by the Jacob number (8) while the effective Jacob number (19) seems to be more relevant for a translating bubble. This reflects the greater instability of the latter situation.

The stability boundaries in the $\left(P_{A}, U\right)$ plane shown in Figs. 9 and 10 must correspond to conditions such that the rectified influx of heat due to the sound-induced oscillations balances the heat lost through convection. When conditions are such that the bubble lasts many cycles, the heat exchange with the liquid may approximately be considered as governed by a Nusselt number of the form (11) averaged over one cycle. In these conditions since, to lowest order, rectified heat flux into the bubble is a second order effect and, therefore, proportional to $P_{A}^{2}$ (see, e.g., Wang, 1974; Gumerov, 2000), one may expect from (11) a dependence of the stability boundary on velocity of the form $P_{A}^{2} \propto \sqrt{U}$, i.e., $P_{A}$ $\propto U^{1 / 4}$. Figures 17 and 18 show the same results as Figs. 9 and 10 replotted in this way. At very low translational velocity, conduction dominates, the threshold becomes independent of $U$ and, therefore, $P_{A} / U^{1 / 4}$ diverges. To explain the high-velocity behavior of some of the curves, we observe that proportionality of rectified heat transfer to $P_{A}^{2}$ can only be assumed when the bubble lasts long enough to take time averages. If the bubble only lasts a few cycles, this estimate is inapplicable and, indeed, we see in Figs. 17 and 18 that, for some frequencies, the proportionality of $P_{A}$ to $U^{1 / 4}$ is violated at higher velocities. Nevertheless, we see that there are velocity and frequency ranges where the prediction $P_{A}$ $\propto U^{1 / 4}$ is substantiated by the numerical results.

It is evident from many of the examples shown in the previous figures that the rectified diffusion of heat into the bubble becomes much smaller past the resonance radius. It is therefore conceivable that a bubble for which rectified diffusion is just about strong enough to make it grow below resonance would start to condense past resonance then grow again once it has shrunk below the resonance radius, and so on. We have searched numerically for this behavior but have concluded that, if it exists, it requires such a fine tuning of conditions as to be of little practical interest; therefore, we have not pursued the matter further.

\section{CONCLUSIONS}

In view of the dependence of heat transfer on convection, it is not surprising to find a strong effect of translatory

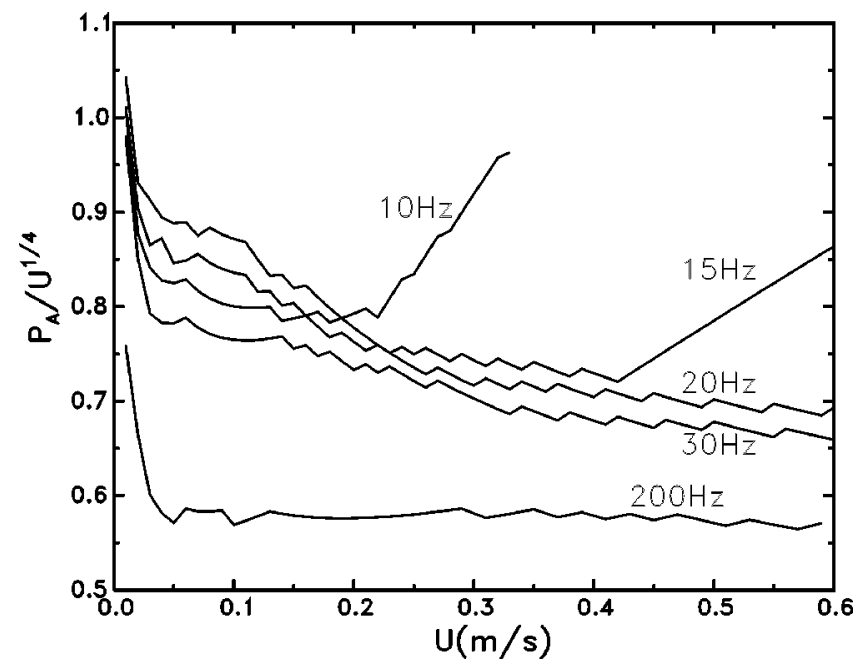

FIG. 17. The data of Fig. $9[R(0)=0.5 \mathrm{~mm}]$ replotted as $P_{A} / U^{1 / 4}$ vs $U$ according to the argument given at the end of Sec. IV. The horizontal or near-horizontal portion of the curves corresponds to situations in which convection dominates the heat transfer from the bubble. The curves are labeled by the sound frequency in $\mathrm{Hz}$. 


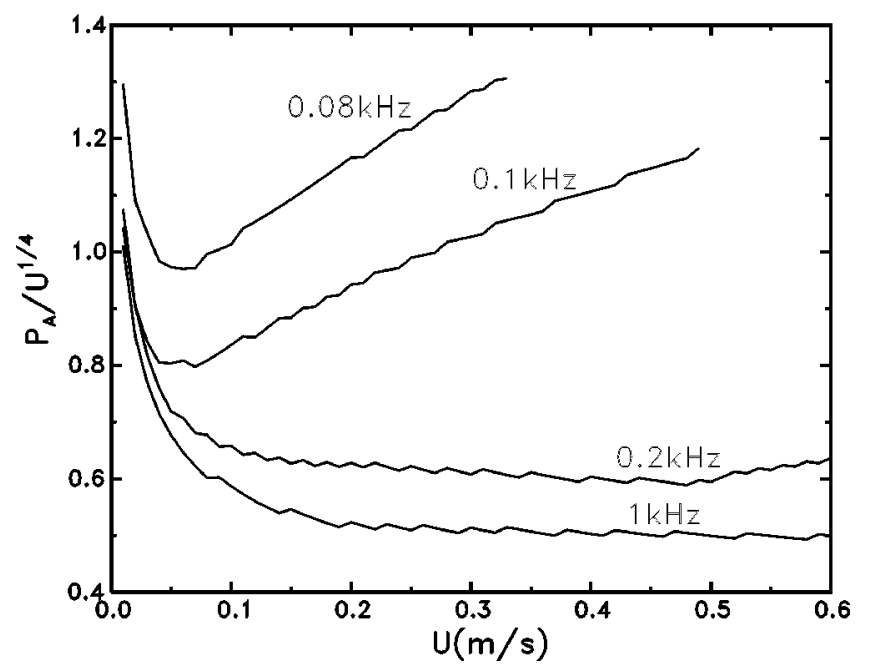

FIG. 18. The data of Fig. $10[R(0)=0.1 \mathrm{~mm}]$ replotted as $P_{A} / U^{1 / 4}$ vs $U$ according to the argument given at the end of Sec. IV. The horizontal or near-horizontal portion of the curves corresponds to situations in which convection dominates the heat transfer from the bubble. The curves are labeled by the sound frequency in $\mathrm{kHz}$.

motion on the process of rectified heat transfer into a vapor bubble. The details of the process are complex and depend on the initial liquid temperature distribution around the bubble, in addition to the bubble radius, velocity, and sound field characteristics. This circumstance prevents one from making simple statements of general validity. If the liquid is initially subcooled and at a uniform temperature, convection may destabilize the bubble. For example, in water at $95^{\circ} \mathrm{C}$ and at normal gravity, a $1-\mathrm{mm}$-diam bubble in a $1-\mathrm{kHz}$, $30.39-\mathrm{kPa}$ sound field would grow if moving at $0.2 \mathrm{~m} / \mathrm{s}$, while it would collapse at $0.3 \mathrm{~m} / \mathrm{s}$. In a stronger sound field, $35.46 \mathrm{kPa}$, the bubble would be stabilized even at this higher velocity, which is of the order of the buoyant rise velocity of such a bubble in normal gravity. Qualitatively, an interesting finding of this study is that the boundary between stable and unstable bubbles occurs in parameter ranges readily encountered in practice.

These effects may be useful for bubble management in several conditions. For example, in situations where large bubbles may be easier to control than smaller ones, one may want to promote growth, which facilitates coalescence under the action of pressure-radiation forces (Hao et al., 2001). Our results show that this is possible even in the presence of subcooled convection and give an indication as to the necessary acoustic parameters.

\section{ACKNOWLEDGMENTS}

The authors are grateful to Dr. N. Gumerov for some useful exchanges in the course of this work and to the re- viewers for some useful suggestions. This study has been supported by NASA under Grant No. NAG3-1924.

${ }^{1}$ Dr. Gumerov (private communication) points out that an additional mechanism might be the different curvature of the saturation and adiabatic curves: the temperature oscillations have a shift due to the nonlinearity of these curves which causes an effective liquid-vapor temperature difference to arise.

${ }^{2} \mathrm{~A} 0.5$-mm-radius bubble in water has a rise velocity of the order of $0.3 \mathrm{~m} / \mathrm{s}$ under normal buoyancy; larger or smaller velocities are possible due to acceleration of the container, different gravitational fields, the presence of shock waves, and others.

${ }^{3}$ We loosely refer to a bubble prevented from collapsing as being "stabilized" by the action of the sound field. The true equilibrium state corresponds to a condition in which mass loss by condensation balances gain by rectified diffusion; it is clear, however, that this equilibrium is unstable.

Akulichev, V. A., Alekseev, V. N., and Yushin, V. P. (1979). "Growth of vapor bubbles in an ultrasonic field,' Sov. Phys. Acoust. 25, 453-457.

Ervin, J. S., Merte, H., Jr., Keller, R. B., and Kirk, K. (1992). "Transient pool boiling in microgravity," Int. J. Heat Mass Transf. 35, 659-674.

Florschuetz, L. W., and Chao, B. T. (1965). "On the mechanics of vapor bubble collapse," J. Heat Transfer 87, 209-220.

Fyrillas, M. M., and Szeri, A. J. (1994). "Dissolution or growth of soluble spherical oscillating bubbles," J. Fluid Mech. 277, 381-407.

Gumerov, N. A. (2000). "Dynamics of vapor bubbles with non-equilibrium phase transitions in isotropic acoustic fields," Phys. Fluids 12, 71-88.

Hao, Y., and Prosperetti, A. (1999). "The dynamics of vapor bubbles in acoustic pressure fields," Phys. Fluids 11, 2008-2019.

Hao, Y., and Prosperetti, A. (2000). "The collapse of vapor bubbles in a spatially non-uniform flow,' Int. J. Heat Mass Transf. 43, 3539-3550.

Hao, Y., Oğuz, H. N., and Prosperetti, A. (2001). "Pressure-radiation forces on pulsating vapor bubbles," Phys. Fluids 13, 1167-1177.

Khabeev, N. S. (1976). "Heat transfer and phase-transition effects in the oscillation of vapor bubbles," Sov. Phys. Acoust. 21, 501-505.

Legendre, D., Borée, J., and Magnaudet, J. (1998). “Thermal and dynamic evolution of a spherical bubble moving steadily in a superheated or subcooled liquid," Phys. Fluids 10, 1256-1272.

Oka, T., Abe, Y., Tanaka, K., Mori, Y. H., and Nagashima, A. (1992). “Observational study of pool boiling under microgravity," JSME Int. J.: Series B 35, 280-286.

Patel, G. M., Nicholas, R. E., and Finch, R. D. (1985). "Rectified heat transfer in vapor bubbles," J. Acoust. Soc. Am. 78, 2122-2131.

Prosperetti, A., and Hao, Y. (2001). "Vapor bubbles in flow and acoustic fields," in Free-Surface Flows, edited by A. C. King and Y. Shikhmurzaev (Kluwer, Dordrecht), pp. 249-256.

Prosperetti, A., and Hao, Y. (2002). "Vapor bubbles in flow and acoustic fields," in Annals of the New York Academy of Sciences, vol. 974, Microgravity Transport Processes in Fluid, Thermal, Biological, and Materials, Sciences, edited by S. S. Sadhal, in press.

Ruckenstein, E. (1959). "On heat transfer between vapour bubbles in motion and the boiling liquid from which they are generated," Chem. Eng. Sci. 10, 22-30.

Sitter, J. S., Snyder, T. J., Chung, J. N., and Marston, P. L. (1998a). “Acoustic field interaction with a boiling system under terrestrial gravity and microgravity," J. Acoust. Soc. Am. 104, 2561-2569.

Sitter, J. S., Snyder, T. J., Chung, J. N., and Marston, P. L. (1998a). "Terrestrial and microgravity pool boiling heat transfer from a wire in an acoustic field," Int. J. Heat Mass Transf. 41, 2143-2155.

Wang, T. (1974). "Rectified heat transfer," J. Acoust. Soc. Am. 56, 11311143 . 